\title{
ICU Risk Stratification Models Feasible for Use in Sub-Saharan Africa Show Poor Discrimination in Malawi: A Prospective Cohort Study
}

\author{
Meghan $\operatorname{Prin}^{1} \cdot$ Stephanie Pan $^{2} \cdot$ Clement Kadyaudzu ${ }^{3} \cdot$ Guohua Li $^{4,5} \cdot$ Anthony Charles $^{6}$
}

Published online: 16 July 2019

\begin{abstract}
Background Critical illness disproportionately affects people in low-income countries (LICs). Efforts to improve critical care in LICs must account for differences in demographics and infrastructure compared to high-income settings. Part of this effort includes the development and validation of intensive care unit (ICU) risk stratification models feasible for use in LICs. The purpose of this study was to validate and compare the performance of ICU mortality models developed for use in sub-Saharan Africa.

Materials and Methods This was a prospective, observational cohort study of ICU patients in a referral hospital in Malawi. Models were selected for comparison based on a Medline search for studies which developed ICU mortality models based on cohorts in sub-Saharan Africa. Model discrimination was evaluated using the area under the curve with $95 \%$ confidence intervals (CI).

Results During the study, 499 patients were admitted to the study ICU, and after exclusions, there were 319 patients. The cohort was $62 \%$ female, with the mean age 31 years (IQR: 23-41), and $74 \%$ had surgery preceding ICU admission. Discrimination for hospital mortality ranged from $0.54(95 \%$ CI $0.48,0.60)$ for the Universal Vital Assessment (UVA) to 0.72 (95\% CI 0.66, 0.78) for the Malawi Intensive care Mortality Evaluation (MIME). After tenfold cross-validation, these results were unchanged.

Conclusions The MIME outperformed other models in this prospective study. Most ICU models developed for LICs had poor to modest discrimination for hospital mortality. Future research may contribute to a better risk stratification model for LICs by refining and enhancing the MIME.
\end{abstract}

Part of this work was presented as a poster at the 2018 American Society of Anesthesiology annual meeting in San Francisco, California, USA.

Electronic supplementary material The online version of this article (https://doi.org/10.1007/s00268-019-05078-9) contains supplementary material, which is available to authorized users.

Meghan Prin

prin@bcm.edu; meghan.prin@gmail.com

Stephanie Pan

Stephanie.pan6@gmail.com

Clement Kadyaudzu

kadyaudzu@yahoo.com

\section{Introduction}

Critical illness disproportionately affects people in lowincome countries (LICs) of sub-Saharan Africa [1]. Providing quality intensive care medicine has the potential to mitigate preventable mortality in these settings, but

Guohua Li

g12240@cumc.columbia.edu

Anthony Charles

anthony_charles@med.unc.edu 
extrapolating intensive care medicine protocols and strategies to LICs is challenging. Efforts to improve intensive care medicine in LICs must account for differences in the patient demographics, clinical case-mix, and healthcare system infrastructure. A necessary component of this enterprise is the development and validation of intensive care unit (ICU) risk stratification models feasible for use in LICs.

Models developed specifically for LICs are necessary because existing models developed in high-income settings (e.g., Acute Physiologic Assessment and Chronic Health Evaluation [2], Mortality Prediction Models [3], Simplified Acute Physiology Score [4]) have not shown strong discrimination when applied to LIC populations [5]. Furthermore, these models rely on advanced or multiple laboratory assessments (e.g. arterial blood gas) which are not usually available in LICs. Several studies have recently put forth ICU risk models and early-warning scores feasible for use to assess critically ill patients in sub-Saharan Africa [6-10]. None of these models have been externally validated by an independent study. The purpose of this study was to validate and compare the discrimination of these models for hospital and ICU mortality, using a prospective ICU cohort in Malawi.

\section{Methods}

This was a prospective, observational cohort study of patients admitted to the ICU of Kamuzu Central Hospital $(\mathrm{KCH})$ in Lilongwe, Malawi, from September 2016 to July 2018. The anticipated sample size and study time period was based on other similar studies [7]. The study protocol was registered at researchregistry.com under protocol 4330. It was developed a priori and approved by the National Health Sciences Research Council of Malawi and the institutional review boards of both American universities with which the study was affiliated, and the requirement for written informed consent was waived by all.

1 Department of Anesthesiology, Baylor College of Medicine, 1 Baylor Plaza, Houston, TX 77030, USA

2 Icahn School of Medicine At Mt. Sinai, New York, NY, USA

3 Department of Anesthesiology, Kamuzu Central Hospital, Lilongwe, Malawi

4 Department of Anesthesiology, Columbia University College of Physicians \& Surgeons, New York, NY, USA

5 Department of Epidemiology, Columbia University Mailman School of Public Health, New York, NY, USA

6 Department of Surgery, University of North Carolina At Chapel Hill, Chapel Hill, NC, USA
Malawi is a country in southern Africa with a population of 18 million people, a life expectancy of 63.8 years, and a Human Development Index rank of 170 out of 187 countries [11]. It is the sixth poorest country in sub-Saharan Africa [12]. $\mathrm{KCH}$ is a referral hospital in the central region of Malawi with a catchment area of approximately 5 million. The ICU at $\mathrm{KCH}$ is a five-bed unit which offers a 1:1 nurse-to-patient ratio, continuous noninvasive vital sign monitoring, mechanical ventilation with a titratable fraction of inhaled oxygen, and intravenous medication infusions. Hemodialysis is available within the hospital if patients can be transferred to the nearby unit where it is provided. Clinical care in the ICU is directed by clinical officers in anesthesiology. No staff members have consultant-level expertise in intensive care medicine.

All patients admitted to the study ICU were eligible for inclusion. Exclusion criteria included age $\leq 15$ years, readmission to ICU, and ICU admission for a reported severe head injury. Supplemental analyses included patients with a reported severe head injury, to assess the validity of the predictive models in the larger cohort. The primary outcome was hospital mortality. The secondary outcome was ICU mortality.

The data were collected prospectively by study staff trained in ICU data abstraction. Study staff began data collection for each patient at the time of ICU admission by reviewing the medical files and then following the patient through his or her hospital course. Variables collected included date of hospital admission, hospital location before ICU admission (e.g., emergency room, operating room, ward), the admitting service (e.g., surgery, obstetrics and gynecology, medicine, pediatrics), vital signs and laboratory measurements at the time of admission to ICU, treatments utilized during the ICU admission (e.g., mechanical ventilation), the location to which patients were discharged, and the hospital discharge date. Laboratory measurements at the time of ICU admission included only those commonly measured by local clinical standards and readily available in the hospital laboratory, rather than more expensive tests frequently used in high-income settings. For this study, a complete blood count, electrolytes, point-of-care malaria status, and point-of-care human immunodeficiency virus (HIV) status were included. Vital sign measurement included an assessment of mental status using the AVPU scale (alert, verbally responsive, painfulstimulus responsive, and unresponsive), which was simpler and more acceptable to local clinicians than the Glasgow Coma Scale scoring system. However, because this variable was frequently confounded by postoperative residual anesthesia, during data analysis, we simplified it into an assessment of altered mental status (i.e., alert versus any value other than alert). We also recorded the clinical suspicion of infection at ICU admission and the presence of 
Table 1 ICU risk models and early-warning scores feasible for use in low-income settings and selected for comparison

\begin{tabular}{|c|c|}
\hline Model & $\begin{array}{l}\text { Geographic location of } \\
\text { development cohort }\end{array}$ \\
\hline $\begin{array}{l}\text { Malawi intensive care mortality risk } \\
\text { evaluation model (MIME) [6] }\end{array}$ & Malawi \\
\hline Simplified MIME (sMIME) & Malawi \\
\hline $\begin{array}{l}\text { Modified Early Warning Score } \\
\text { (MEWS) [8] }\end{array}$ & UK \\
\hline $\begin{array}{l}\text { Simplified Rwanda-mortality } \\
\text { prediction model (sR-MPM) [7] }\end{array}$ & Rwanda \\
\hline $\begin{array}{c}\text { Quick Sequential Organ Failure } \\
\text { Assessment (qSOFA) [9] }\end{array}$ & $\begin{array}{l}\text { International task force } \\
\text { recommendation }\end{array}$ \\
\hline $\begin{array}{l}\text { Universal Vital Assessment (UVA) } \\
\text { [10] }\end{array}$ & $\begin{array}{c}\text { Gabon, Malawi, Sierra Leone, } \\
\text { Tanzania, Uganda, Zambia }\end{array}$ \\
\hline Modified Universal Vital Assessment & $\begin{array}{c}\text { Gabon, Malawi, Sierra Leone, } \\
\text { Tanzania, Uganda, Zambia }\end{array}$ \\
\hline
\end{tabular}

fever $\left(>38.4{ }^{\circ} \mathrm{C}\right.$ ) at any time during the ICU course. All records were initially kept on paper and then in a deidentified computer database.

The models chosen for comparison were selected based on a Medline search for studies published within the past 10 years which developed mortality prediction models for critically ill hospitalized patients based on cohorts in subSaharan Africa. We focused on a framework of critically ill hospitalized patients, rather than specifically on ICU patients, given the understanding that many hospitals in the region either do not have ICU facilities or have a very small ICU facility to which all critically ill patients do not have access. We also included models like the quick Sequential Organ Failure Assessment (qSOFA) [9] and Modified Early Warning Score (MEWS) [8], which were not developed for mortality modeling per se, but which have been evaluated within sub-Saharan Africa hospital cohorts in recent studies $[13,14]$. In some cases, we were only able to apply simplified or modified versions of models based on the ability to collect clinical information at our study site; for example, Glasgow Coma Scale (GCS) scoring is not easily performed at our study site and there was a high proportion of missing data in this variable. As a result, we were able to evaluate the simplified Rwanda Mortality Prediction Model but not the full Rwanda Mortality Prediction Model. For the same reason, we modified the Universal Vital Assessment (UVA) by substituting the AVPU scale for GCS scores (Supplement, Table 1,2) The final models selected for comparison are summarized in Table 1.

We described and assessed the association of the component variables for each model with hospital and ICU mortality using univariable and multivariable logistic regression. (Supplement, Table 3a-b, 4a-b) Model discrimination was then evaluated using the area under the receiver operating characteristic curve (AUC, or c-statistic) with 95\%
Component variables

Age, sex, admitting service, systolic pressure, mental status, and fever $(>38.4 \mathrm{C}$ ) during the ICU course

ICU admitting service, fever during ICU course ( $>38.4 \mathrm{C})$, mental status

Temperature, heart rate, respiratory rate, systolic blood pressure, and mental status

Age, heart rate, infection, shock or hypotension, mental status

Respiratory rate, systolic blood pressure, and mental status

Temperature, heart rate, respiratory rate, systolic blood pressure, oxygen saturation, Glasgow coma scale, HIV status

Temperature, heart rate, respiratory rate, systolic blood pressure, oxygen saturation, mental status, and HIV status

confidence intervals (CI). The area under the curve (AUC) summarizes how well a model is able to accurately delineate survival or non-survival following ICU admission. For the MEWS, for which cutoff scores have been suggested, we also evaluated the AUC of different cutoffs [14]. Model performance was assessed using Akaike information criterion (AIC), Bayesian information criterion (BIC), and $R$-squared. Internal validation of the models' accuracy was performed using tenfold cross-validation. In other words, we partitioned the study sample randomly into 10 equal subsets, where each subset is a "fold." For each fold, we designate it as the testing set (10\% of the data) and train on the remaining $90 \%$ of the data. This process is repeated 10 times for each fold, and the AUC, accuracy, sensitivity, specificity, and ROC curves are averaged with the range and standard deviations reported. This process was repeated across the seven models. Finally, we performed a comparison of the receiver operating curves across all the models using the nonparametric approach of DeLong, DeLong, and Clarke-Pearson [15]. For each of the scoring methods, the $\mathrm{p}$ value is computed from the pair-wise comparison of AUC against the MIME method; AUC differences and $95 \%$ confidence interval around that difference are also reported. In this comparison, a cutoff of 6 was chosen for the MEWS model based on preceding analyses. Statistical analyses were conducted using SAS Version 9.4 (SAS Institute, Inc., Cary, NC). The results are reported in line with the Strengthening the Reporting of Cohort Studies in Surgery (STROCSS) criteria [16].

\section{Results}

During the study period, 499 patients were admitted to the study ICU. After excluding for age $\leq 15$ years and readmission $(n=103)$, there were 396 patients. After excluding 
Table 2 Demographics and clinical traits of study population

Baseline variables and clinical characteristics

Male sex, $n(\%)$

Age, median (IQR)

ICU admitting service, $n(\%)$

General surgery

Obstetrics and gynecology

Medicine

Location before ICU admission, $n(\%)$

General ward

High-dependency unit ${ }^{\mathrm{b}}$

Emergency room

Operating theater

Transfer from outside hospital

Surgery in $72 \mathrm{~h}$ preceding ICU admission, $n(\%)$

Vital signs at admission to ICU

Heart rate (bpm), median (IQR)

Respiratory rate, median (IQR)

Mean arterial pressure ${ }^{\mathrm{c}}(\mathrm{mmHg})$, median (IQR)

Temperature (C), median (IQR)

Pulse oximetry value, median (IQR)

Suspected of having infection at admission to ICU, $n(\%)$

HIV positive, $n(\%)$

Malaria status positive, $n(\%)$

Provision of mechanical ventilation, $n(\%)$

Length of mechanical ventilation, days, median (IQR)

Length of stay in ICU, days, median (IQR)

ICU Mortality, $n(\%)$
All patients $(n=319)$

\begin{tabular}{|c|c|c|c|c|c|}
\hline \multicolumn{2}{|c|}{$\begin{array}{l}\text { Survived hospital } \\
(n=130)\end{array}$} & \multicolumn{2}{|c|}{$\begin{array}{l}\text { Died in hospital } \\
(n=174)\end{array}$} & \multicolumn{2}{|c|}{ Total $(n=319)^{\mathrm{a}}$} \\
\hline$N$ & $\begin{array}{l}\text { Median (IQR) } \\
\text { or } N(\%)\end{array}$ & $N$ & $\begin{array}{l}\text { Median (IQR) } \\
\text { or } N(\%)\end{array}$ & $N$ & Median (IQR) or $N(\%)$ \\
\hline 130 & $43(33)$ & 174 & $75(43)$ & 319 & $121(38)$ \\
\hline 130 & $30.5(23-37)$ & 174 & $32.5(23-43)$ & 319 & $31(23-41)$ \\
\hline 130 & $45(35)$ & 174 & $87(50)$ & 319 & $139(44)$ \\
\hline 130 & $51(39)$ & 174 & $40(23)$ & 319 & $98(31)$ \\
\hline 130 & $34(26)$ & 174 & $47(27)$ & 319 & $82(26)$ \\
\hline 127 & $10(8)$ & 168 & $7(4)$ & 310 & $18(6)$ \\
\hline 127 & $29(23)$ & 168 & $41(24)$ & 310 & $75(24)$ \\
\hline 127 & $12(10)$ & 168 & $17(10)$ & 310 & $29(10)$ \\
\hline 127 & $71(56)$ & 168 & $97(58)$ & 310 & $177(57)$ \\
\hline 127 & $5(4)$ & 168 & $6(4)$ & 310 & $11(4)$ \\
\hline 130 & $96(74)$ & 173 & $128(74)$ & 318 & $234(74)$ \\
\hline 128 & $120(100-133)$ & 170 & $119(102-136)$ & 313 & $119(102-135)$ \\
\hline 125 & $19(15-25)$ & 163 & $20(14-26)$ & 303 & $20(15-26)$ \\
\hline 128 & $88.4(72-105)$ & 169 & $82.2(61-99)$ & 312 & $85.8(68-101)$ \\
\hline 121 & $36(35-37)$ & 161 & $36(34-37)$ & 297 & $36(34-37)$ \\
\hline 124 & 98 (94-99) & 152 & 97 (91-99) & 290 & 97 (92-99) \\
\hline 130 & $83(64)$ & 173 & $112(65)$ & 317 & $201(63)$ \\
\hline 113 & $9(8)$ & 142 & $28(20)$ & 266 & 37 (14) \\
\hline 100 & $10(10)$ & 128 & $16(13)$ & 241 & $29(12)$ \\
\hline 130 & $119(92)$ & 172 & $170(99)$ & 316 & $302(96)$ \\
\hline 116 & $2(1-3)$ & 171 & $2(1-4)$ & 297 & $2(1-4)$ \\
\hline 129 & $3(2-4)$ & 170 & $2(1-5)$ & 312 & $2.5(1-5)$ \\
\hline 130 & $0(0)$ & 174 & $154(89)$ & 315 & $154(49)$ \\
\hline
\end{tabular}

aThere were 15 patients with an unknown status for hospital mortality which are not included in the first two columns bOne of the four hospital high-dependency units (HDUs), including surgical HDU, obstetric HDU, medical HDU, and pediatric HDU cMean arterial pressure is calculated by: 0.66 (diastolic blood pressure) +0.33 (systolic BP)

$B p m$ beats per minute; $I Q R$ interquartile range; $I C U$ intensive care unit; $m m H g$ millimeters of Mercury; $C$ Celcius; $H I V$ human immunodeficiency virus

those with severe head injuries, there were 319 patients. (For analyses including patients with severe head injuries, please refer to the Supplement.) Fifteen patients were missing hospital outcome data and were excluded from analysis of the primary outcome hospital mortality; they are included in descriptive statistics and analysis of the secondary outcome: ICU mortality. The cohort was $62 \%$ female, and the median age was 31 years (interquartile range, IQR, 23-41). The cohort was largely surgical; 74\% of patients had surgery in the $72 \mathrm{~h}$ preceding ICU admission, and $57 \%$ were admitted directly from the operating theater (Table 2).
In the initial analysis, model discrimination for hospital mortality ranged from $0.54(95 \%$ CI $0.48,0.60)$ for UVA to $0.72(95 \%$ CI $0.66,0.78)$ for MIME, and discrimination for ICU mortality ranged from 0.55 (95\% CI $0.49,0.61)$ for UVA to 0.72 (95\% CI 0.66, 0.78) for MIME. (Table 3, Supplement Table 5) After tenfold cross-validation, these results were unchanged. (Figs. 1 and 2). We have sufficient evidence that at least one of the scoring models differs from another scoring method for hospital mortality $(p=0.002)$ and ICU mortality $(p<0.001)$; pair-wise comparisons with the MIME scoring method are shown in Supplement Table 6. 
Table 3 Model discrimination, fit, and internal validation for predicting hospital mortality after tenfold cross-validation

\begin{tabular}{|c|c|c|c|c|c|c|c|}
\hline Model discrimination and fit & MIME & sMIME & sR-MPM & MEWS $^{\mathrm{a}}$ & qSOFA & UVA & $\begin{array}{l}\text { Modified } \\
\text { UVA }\end{array}$ \\
\hline \multirow{2}{*}{$\begin{array}{l}\text { AUC }(0-1 \text {, higher indicates better prediction }) \\
\quad(95 \% \mathrm{CI})\end{array}$} & 0.72 & 0.70 & 0.67 & 0.61 & 0.64 & 0.54 & 0.60 \\
\hline & $\begin{array}{c}(0.66 \\
0.78)\end{array}$ & $\begin{array}{c}(0.64, \\
0.76)\end{array}$ & $\begin{array}{c}(0.61 \\
0.73)\end{array}$ & $\begin{array}{c}(0.56, \\
0.66)\end{array}$ & $\begin{array}{c}(0.58, \\
0.71)\end{array}$ & $\begin{array}{c}(0.48, \\
0.60)\end{array}$ & $(0.54,0.65)$ \\
\hline AIC (lower indicates better model fit) & 374.35 & 375.27 & 386.65 & 375.66 & 378.88 & 419.07 & 408.79 \\
\hline BIC (lower indicates better model fit) & 403.79 & 393.70 & 408.77 & 382.97 & 393.50 & 430.22 & 419.94 \\
\hline $\mathrm{R}^{2}(0-1$, higher indicates better prediction) & 0.13 & 0.12 & 0.09 & 0.06 & 0.07 & 0.01 & 0.04 \\
\hline \multicolumn{8}{|l|}{$\begin{array}{l}\text { Performance on tenfold cross-validated testing } \\
\text { cohort }\end{array}$} \\
\hline Accuracy, $\%$, mean (SD) & $\begin{array}{l}62.3 \\
\quad(10.6)\end{array}$ & $65.1(9.5)$ & $63.8(7.8)$ & $63.7(9.5)$ & $63.6(7.6)$ & $53.4(7.3)$ & $61.8(7.3)$ \\
\hline Sensitivity, $\%$, mean (SD) & $77.9(13)$ & $\begin{array}{l}81.3 \\
\quad(10.8)\end{array}$ & $\begin{array}{l}83.5 \\
\quad(10.3)\end{array}$ & $\begin{array}{l}82.9 \\
\quad(10.1)\end{array}$ & $88.3(8.1)$ & $90.9(15)$ & $77.9(14.3)$ \\
\hline Specificity, $\%$, mean (SD) & $\begin{array}{l}41.5 \\
\quad(11.8)\end{array}$ & $43.5(12)$ & $\begin{array}{l}37.9 \\
\quad(12.2)\end{array}$ & $\begin{array}{l}41.1 \\
\quad(19.1)\end{array}$ & $\begin{array}{l}30.3 \\
\quad(12.5)\end{array}$ & $5.5(10.1)$ & $41(12.8)$ \\
\hline \multirow[t]{2}{*}{ AUC, mean (min, max) } & 0.72 & 0.70 & 0.67 & 0.61 & 0.64 & 0.54 & 0.60 \\
\hline & $\begin{array}{c}(0.70 \\
0.73)\end{array}$ & $\begin{array}{c}(0.68 \\
0.72)\end{array}$ & $\begin{array}{c}(0.66 \\
0.68)\end{array}$ & $\begin{array}{c}(0.59 \\
0.63)\end{array}$ & $\begin{array}{c}(0.63 \\
0.65)\end{array}$ & $\begin{array}{c}(0.52 \\
0.56)\end{array}$ & $(0.58,0.61)$ \\
\hline
\end{tabular}

a.MEWS $\geq 6$

AIC Akaike information criterion; $A U C$ area under the receiver operating curve; BIC Bayesian information criterion; $S D$ standard deviation; MEWS Modified Early Warning Score; MIME Malawi Intensive care Mortality risk Evaluation; qSOFA quick Sequential Organ Failure Assessment; $s-M I M E$ simplified Malawi Intensive care Mortality risk Evaluation; $s R-M P M$ simplified Rwanda Mortality Prediction Model; UVA Universal Vital Assessment

In Supplementary Analyses which included patients with severe head injuries, descriptive analyses of the model components stratified by hospital and ICU mortality are summarized in Supplement Table 7a-b, and assessments of their associations with hospital and ICU mortality are summarized in Supplemental Table 8a-b. Model discrimination for hospital mortality ranged from 0.53 (95\% CI $0.48,0.58)$ for the UVA to $0.70(95 \%$ CI $0.65,0.75)$ for the MIME, and for ICU mortality, it ranged from 0.53 (95\% CI $0.48,0.59)$ for the UVA to $0.70(95 \%$ CI $0.65,0.75)$ for the MIME. These results were unchanged after tenfold crossvalidation. (Supplement Table 9a-b, Supplement Figures 1 and 2). In these analyses, there was also evidence that at least one of the models differ from another scoring method for hospital mortality $(p=0.002)$ and ICU mortality $(p<0.001)$ (Supplement Table 10).

\section{Discussion}

In this prospective cohort study of critically ill patients admitted consecutively to the ICU of a referral hospital in Malawi, we found that seven risk stratification models feasible for use in low-resource ICU settings had poor to moderate discrimination and calibration. These results are not as discouraging as they initially appear. First, the relative simplicity of these models hints at the known trade- off between data collection burden and prognostic precision [17]. Modeling outcomes for a heterogeneous ICU population with complex critical illness using a collection of vital signs will not have the same precision as models with more component variables such as the APACHE. Second, all the models in this analysis are based on relatively small derivation cohorts. Despite the rigor of the studies on which these models are based, these sample sizes reflect the inherent difficulties of collecting prospective clinical data within the LICs of sub-Saharan Africa and underlines the importance of investing in data collection systems which may allow for the creation of larger clinical registries in this region. Finally, these models are remarkably similar, relying in large part upon the same basic vital signs (blood pressure, temperature, mental status), which contributes to the consistently poor to moderate performance for each model within this cohort.

In evaluating the performance of these models, physicians and public health workers must also recognize that not all ICUs within LICs are the same. For example, the cohort upon which the MIME was based was $74 \%$ surgical, $62 \%$ female, and had an HIV prevalence of $15 \%$ [6]. In contrast, the cohort upon which the simplified RwandaMPM was based included $23 \%$ surgical patients and $51 \%$ female patients, and $5 \%$ had an HIV prevalence [7]. The UVA model was based on a cohort in which $38 \%$ of subjects were HIV positive. While the study which associated 

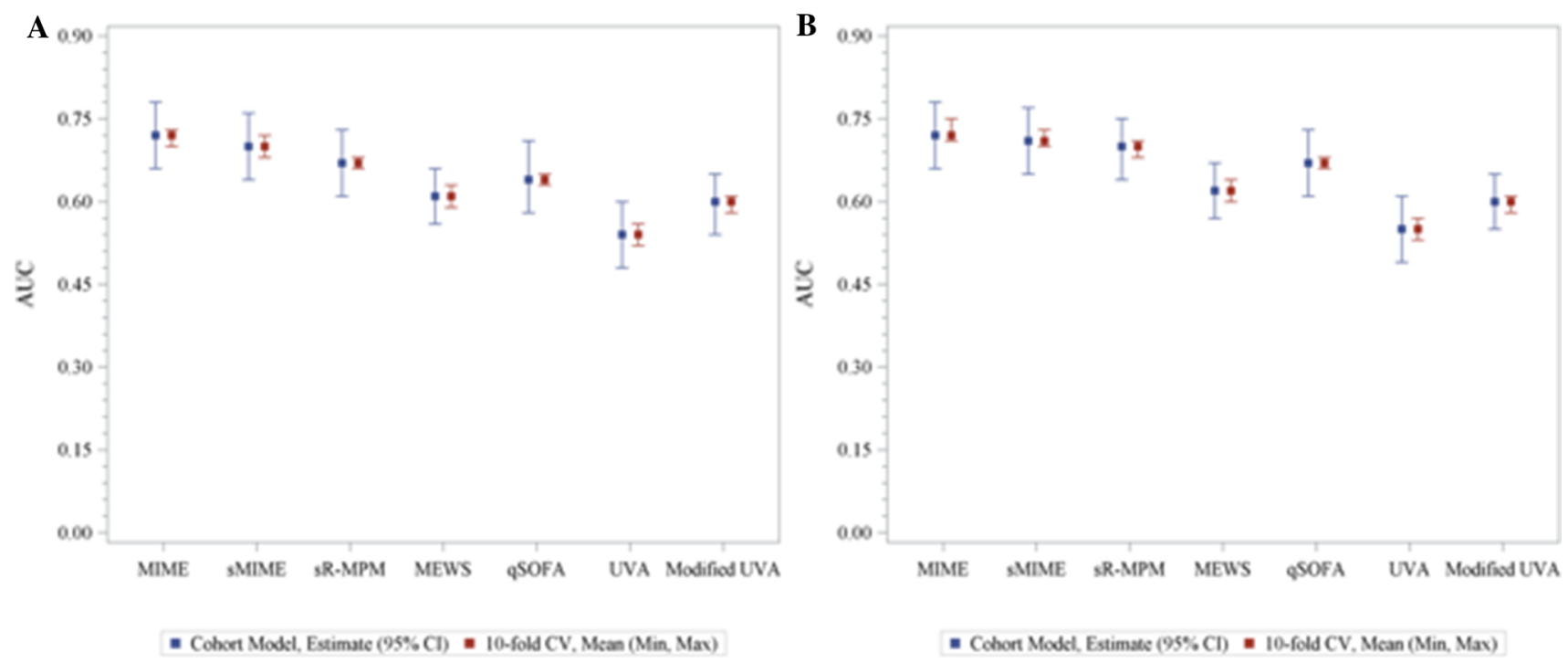

Fig. 1 Comparison of AUC across all scoring methods
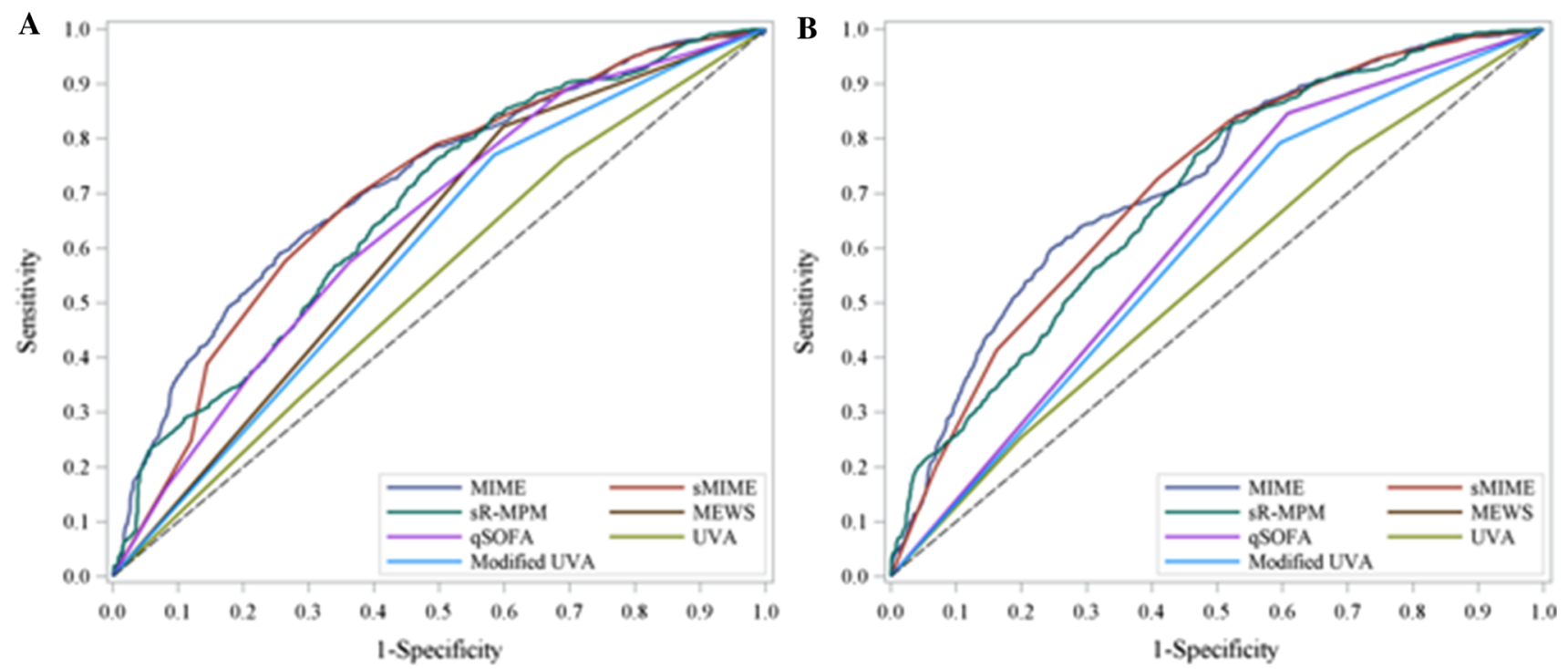

Fig. 2 Averaged tenfold cross-validated ROC curve compared across all scoring methods

MEWS with critical illness in Uganda included a large proportion of surgical patients (55\%), it also had a higher HIV prevalence (24\%) than the MIME or Rwanda-MPM cohorts [14]. Attempting to derive and fit the same risk stratification models in these different ICU populations may explain the poor discrimination found in this study.

Furthermore, ICUs in LICs are most often general units, as opposed to the specialized cardiac care or neonatal units found in high-income settings. In the context of a healthcare system that lacks specialized units, ICU medicine may be even more heterogeneous than the 'general' ICUs found in high-income countries. Risk stratification models based on vital signs appear to be insufficient to precisely riskstratify this heterogeneous population. This is evidenced in part through revisiting the MIME derivation cohort: In the initial analysis which excluded head injury patients, the AUC was 0.70 (95\% CI 0.63, 0.76). When severe head injury patients were included in the supplementary analysis, which increased the size and heterogeneity of the cohort, the discrimination of the model did not improve (AUC $0.69,95 \%$ CI 0.63, 0.75). We may develop better models for LICs by focusing on more specific groups of 
patients. This could be achieved with an Obstetric Critical Illness Score versus a Head Injury Critical Illness Score, for example.

This study reiterates the value and importance of clinical registries in advancing critical care medicine in LICs. Advocates for elevating critical care medicine on the global health agenda are constrained by a lack of data from LICs in general and from the sub-Saharan African region in particular. Only good data can inform good critical care delivery and healthcare policy. While the Intensive Care Over Nations (ICON) study purported to study the global burden of critical illness, only 141 patients in this 10,069 patient study (1.4\%) were from Africa [18]. While the ongoing Global Burden of Disease study includes data from all LICs and from across sub-Saharan Africa, it is broad and not designed for patient-oriented critical care research. Detailed, high-quality clinical registries could inform critical care research and the development of stronger risk stratification models, but they will require multilevel investment from government officials and clinicians.

Estimates from the World Bank indicate that by 2030, more than $90 \%$ of the people living in extreme poverty will be in sub-Saharan Africa [19]. As this shift occurs, demand for critical care services within this region can be expected to increase. In order to meet this demand, current research and care models may have to be reconsidered. For example, future ICU risk stratification model development research in LICs may benefit by incorporating social determinants of health which may disproportionately impact critical illness severity and outcomes in low-income contexts: distance from a hospital facility, nutrition history, and family or community support structures for example. These data have been associated with critical illness mortality in high-income settings. For example, Seymour et al. [20] analyzed over 1.1 million hospitalizations for sepsis in the USA and found that single and divorced men and single women have higher hospital mortality than married counterparts. Metersky et al. [21] analyzed over 48,000 elderly male hospitalizations for pneumonia and also found higher mortality among unmarried subjects. Several studies have linked hospitalization rates and outcomes to a patient's geography or distance from the hospital [22-24]. Furthermore, anthropometric assessments such as the Subjective Global Assessment and mid-arm circumference have recently performed better than biochemical markers or the APACHE score, respectively, in predicting hospital outcome among critically ill patients $[25,26]$. These data are relatively easy to collect in low-resourced settings and may add needed precision to current risk stratification models.

Another potential solution to improving ICU risk stratification models is to focus on data which reflect the infrastructure of healthcare in LICs of sub-Saharan Africa. These data may include the nurse-to-patient ratio in the
ICU, intensivist oversight, the ICU census at the time of a new patient's admission, the time of day or the day of the week on which a patient is admitted to ICU, and the availability of key resources (e.g., blood transfusions, hemodialysis, radiologic imaging). Many of these factors have been rigorously evaluated and associated with hospital mortality in high-income settings [27-30] and may add precision to risk stratification models. Anesi et al. [31] recently published a two-center study from South Africa which showed an association between ICU census and ICU mortality. This is one of the first such reports from subSaharan Africa and future studies should attempt to replicate these results.

There are several limitations to this study. First, it is a single-center study. Critical care medicine is still developing as a specialty in many sub-Saharan African countries and is generally only available at referral hospitals. Future studies may benefit from aggregating data from several referral hospitals to increase statistical power and generalizability. Second, some of the data used to validate and compare these models was also used in the development of the MIME, and this may have contributed to the superior performance of the MIME.

\section{Conclusions}

In this prospective cohort study, we found that seven models developed for evaluating critically ill patients in LICs show poor to moderate discrimination. This may be attributable to heterogeneous derivation cohorts or overly simplistic models, but highlights the need for renewed interest and a novel approach for risk stratification of critically ill patients in LICs.

Acknowledgements This study was supported in part by the National Institute of Health Fogarty International Center Postdoctoral Research Fellowship to Dr. Prin. The authors would also like to thank the staff of Kamuzu Central Hospital and UNC-Project Malawi for their support. Thanks also to Dr. Bryan Roller for presenting this work at the American Society of Anesthesiology 2018 annual meeting in San Francisco, CA.

Author contribution MP conceived of the project, contributed to project design, performed data collection and analysis, and helped write the manuscript. SP helped with statistical analysis and manuscript preparation. CK helped with the data collection and to write the manuscript. GL helped with the data collection and to write the manuscript. AC helped conceive of the project, helped with project design, data collection, and to write the manuscript.

\section{References}

1. Murthy S, Adhikari NK (2013) Global health care of the critically ill in low-resource settings. Ann Am Thorac Soc 10(5):509-513 
2. Knaus W, Draper EA, Wagner DP et al (1985) APACHE II: a severity of disease classification system. Crit Care Med 13(10):818-829

3. Lemeshow S, Teres D, Klar J et al (1993) Mortality probability models (MPM II) based on an international cohort of intensive care unit patients. JAMA 270:2478-2486

4. Le Gall JR, Lemeshow S, Saulnier F (1993) A new simplified acute physiology score (SAPS II) based on a European/North American multi center study. JAMA 270:2957-2962

5. Haniffa R, Isaam I, De Silva AP et al (2018) Performance of critical care prognostic scoring systems in low and middle-income countries: a systematic review. Crit Care 22(1):18

6. Prin M, Pan S, Kadyaudzu C et al (2018) Development of a Malawi intensive care mortality risk evaluation (MIME) model, a prospective cohort study. Int J Surg 60:60-66

7. Riviello R, Kiviri W, Fowler R et al (2016) Predicting mortality in low-income country ICUs: The Rwanda mortality probability model (R-MPM). PLoS ONE 11(5):e0155858

8. Stenhouse C, Coates S, Tivey M et al (1999) Prospective evaluation of a modified Early Warning Score to aid earlier detection of patients developing critical illness on a general surgical ward. Br J Anaesth 84:663P

9. Seymour CW, Liu VX, Iwashyna TJ et al (2016) Assessment of clinical criteria for sepsis: for the third international consensus definitions for sepsis and septic shock (Sepsis-3). JAMA 315(8):762-774

10. Moore C, Hazard R, Saulters K et al (2017) Derivation and validation of a universal vital assessment (UVA) score: a tool for predicting mortality in adult hospitalised patients in sub-Saharan Africa. BMJ Glob Health 2:e00344

11. UN: Human Development Reports. United Nations Development Programme 2016 https://hdr.undp.org/en/countries/profiles/MWI Accessed 18 May 2018.

12. J Gregson 2017 The richest countries in the world: International Monetary Fund, World Economic Outlook Database. In: Global Finance

13. Huson MAM, Katete C, Chunda L et al (2017) Application of the qSOFA score to predict mortality in patients with suspected infection in a resource-limited setting in Malawi. Infection 45(6):893-896

14. Kruisselbrink R, Kwizera A, Crowther M et al (2016) Modified early warning score (MEWS) identifies critical illness among ward patients in a resource restricted setting in Kampala, Uganda: a prospective observational study. PLoS ONE 11(3):e0151408

15. DeLong E, Delong D, Clarke-Pearson D (1988) Comparing the areas under two or more correlated receiver operating characteristic curves: a nonparametric approach. Biometrics 44(3):837-845

16. Agha RAB, MR; Vella-Baldacchino, M; Thavayogan R; Orgill DP (2017) STROCCS group: the STROCSS statement: strengthening the reporting of cohort studies in surgery. Int J Surg (in press)
17. Kuzniewicz MW, Vasilevskis EE, Lane R et al (2008) Variation in ICU risk-adjusted mortality: impact of methods of assessment and potential confounders. Chest 133(6):1319-1327

18. Vincent JL, Marshall JC, Namendys-Silva SA et al (2014) Assessment of the worldwide burden of critical illness: the intensive care over nations (ICON) audit. Lancet Respir Med 2(5):380-386

19. Decline of Global Extreme Poverty Continues but Has Slowed: World Bank 2018 Available at https://www.worldbank.org/en/ news/press-release/2018/09/19/decline-of-global-extreme-povertycontinues-but-has-slowed-world-bank Accessed 19 Nov 2018

20. Seymour CI, Iwashyna TJ, Cooke CR, Hough CL, Martin GS (2010) Marital status and the epidemiology and outcomes of sepsis Chest 137(6):1289-1296.

21. Metersky M, Fine M, Mortensen E (2012) The effect of marital status on the presentation and outcomes of elderly male veterans hospitalized for pneumonia. Chest 142(4):982-987

22. O'Meara WP, Noor A, Gatakaa H et al (2009) The impact of primary health care on malaria morbidity-defining access by disease burden. Trop Med Int Health 14(1):29-35

23. Faierman M, Anderson J, Assane A et al (2015) Surgical patients travel longer distances than non-surgical patients to receive care at a rural hospital in Mozambique. Int Health 7(1):60-66

24. Luis A, Cabral P (2016) Geographic accessibility to primary healthcare centers in Mozambique. Int J Equity Health 15:173

25. Hejazi N, Mazloom Z, Zand F et al (2016) Nutritional assessment in critically Ill patients. Iran J Med Sci 41(3):171-179

26. Bector S, Vagianos K, Suh M et al (2016) Does the subjective global assessment predict outcome in critically ill medical patients? J Intensive Care Med 7:485-489

27. Cavallazzi R, Marik P, Hirani A et al (2010) Association between time of admission to the ICU and mortality: a systematic review and metaanalysis. Chest 138(1):68-75

28. Yang S, Wang Z, Liu Z et al (2016) Association between time of discharge from ICU and hospital mortality: a systematic review and meta-analysis. Crit Care 20(1):390

29. Neuraz A, Guérin C, Payet C et al (2015) Patient mortality is associated with staff resources and workload in the ICU: A multicenter observational study. Crit Care Med 43(8):1587-1594

30. Bagshaw S, Wang X, Zygun D et al (2018) Association between strained capacity and mortality among patients admitted to intensive care: A path-analysis modeling strategy. J Crit Care 43:81-87

31. Anesi G, Gabler N, Allorto $\mathrm{N}$ et al (2018) Intensive care unit capacity strain and outcomes of critical illness in a resourcelimited setting: a 2-hospital study in South Africa. J Intensive Care Med. https://doi.org/10.1177/0885066618815804

Publisher's Note Springer Nature remains neutral with regard to jurisdictional claims in published maps and institutional affiliations. 